\title{
Integrating the Image Identifiable Principle of Human Cognition and Computer Vision to Develop a New Pattern Recognition Design System for Smart Home
}

\author{
Pin-Chin Wang, Wan-Ting Tseng, Chun-Min Cheng, Yi-Hsuan Sung, \\ Yi-Chun Chou, and Fong-Gong $\mathrm{Wu}$ \\ Department of Industrial Design, National Cheng Kung University, Tainan 70101, Taiwan \\ \{sa751201, wan3001, min770731\}@gmail.com, \\ lsung.yvonne@yahoo.com.tw, hanako21677@hotmail.com, \\ fonggong@mail.ncku.edu.tw
}

\begin{abstract}
In this study, we invented a new way which classifies objects according to their functions and the regions of use. Then we proceeded to innovate and design the systematic pattern on the objects. For this goal, we make the pattern on the objects as the goal of recognition that can be captured by camera. In this study we intend to design a new way to derive pattern reasonably and that can be recognized by algorithms. The result of our pattern recognition program test pattern that consisted of systematic components showed that the identification rate of success was more than $90 \%$. This result indicated that our pattern design method can derive a significant pattern which is recognized by computer algorithms and can be effective in detecting the state of the object in using.
\end{abstract}

Keywords: Pattern recognition, Smart Home, Kitchen, TUIs.

\section{Introduction}

To use technology to improve our lives, the lifestyle of smart home has generally been considered as the trend of development in the future. Since Ishii and Ullmer [1] have proposed Ubiquitous Computing, this concept flourished rapidly around the world so far[2]. In the environment of smart home, Ubiquitous computing become a practical ideal to improve human life to be more convenient [3]. When a smart home is developed, there should be a thorough system and rules to link users, products, and environments. In order to provide a comfortable environment of smart homes, we aimed to develop a new technology which people can easily access information in the environment and interact with the environment in smart homes.

The purpose of this study is to investigate the integration of ergonomics and human behaviors in smart homes and to make the operation of technology friendlier. In this study we expected to make users interact with system by using tangible user interface (TUIs). The definition of TUIs is the user interface that users interact with a digital system by manipulating physical objects linked to and directly representing some 
aspect of the system[4, 5]. The usage of TUIs can digitize physical objects by computing and represent digital data in physical world, it is the innovative interface that is a two-way linking between physical world and digital world [4]. In this study we expected to use physical objects to interact with system, we attached the recognizable patterns to physical objects so that objects can be recognizing by computer vision. The advantage of using pattern recognition is low cost without additional high-tech accessories on the original object, so users could manipulate objects more natural. Also, it can be used diversely in the smart home system to interact with people. Then we proceeded to innovate and design the systematic pattern on the objects. Finally we make the pattern on the objects as the goal of recognition that can be captured by camera. If the pattern is detected by a machine, it can stimulate a certain action to respond its pattern. According to current the framework of TUIs, in this study we attempt to develop the new technology of pattern recognition that could extend user experience with graphic user interface to operate tangible user interface and breakthrough the limitation of technology. This study focuses on three priorities: (1) Proceeding pattern recognition to detect object, furthermore the pattern design can conform to reasonability. (2) According to the theory of computer vision, we conduct to design the recognizable pattern systematically. (3) We expect this new technology could combine between TUIs and pattern recognition to develop a reasonable and intuitive interface.

\section{$2 \quad$ Literature Review}

\subsection{Tangible User Interface (TUIs)}

Since MIT media Lab have been developed, the tangible user interface (TUI) becomes a new type of interface for the link between digital and physical world. The framework of TUI defined that user use physical objects as operational components to operate and interact with digital data and digital systems [4, 5]. The advantage of Tangible User Interface that the operating range is no longer to limit in the screen, the interface allows users to experience more profound interaction and more close to real life. However, nowadays the development of TUIs encounter some restrictions in usage that lie in setting of control mode[6] and confining space of interaction[7]. Therefore, this study also expect improve these problems with the technology of pattern recognition.

\subsection{Pattern Recognition Technology}

Many researchers predicate home life will be more convenient by information technology[3]. But we must be careful to deal with the link between human behavior and usability of technology. Shin, Jung [8] thought interactivity was the important premise of using the two-dimensional pattern recognition. But there were a few researches investigated how pattern recognition could be applied in tangible interaction. The major scope of pattern recognition that applied in the commercial field, generally well-known one-dimensional bar code and the wide use of the two-dimensional barcode" QR code"[9]. In this study we investigated the usage of 
two-dimensional pattern are somewhat different with barcode, we proposed a concept "augmented interface" that use pattern recognition on TUIs.

\subsection{The Algorithm of Pattern Recognition -- SURF}

In this study we focused the implementation on the algorithm of pattern recognition and pattern design. In many pattern recognition algorithm, Bay, Tuytelaars [10] proposed an algorithm called Speed Up Robust Feature (SURF) that detect interest points on pattern with the feature of scale-invariant and rotation invariant. It improved from Scale-invariant feature transform (SIFT ).SURF algorithm apply widely on the field of image recognition[11]. It allows developers to put pattern model in program to find correspondences between two images of the same scene or object is one of many computer vision applications. It also can be used to develop and test a new pattern design with image recognition. The SURF search for discrete pattern correspondences can be divided into three main features: (1) interest points are selected at distinctive locations in the image, such as corners, cross, blobs, and Tjunctions. The most valuable property of detecting interest point is its repeatability, i.e. whether it reliably finds the same interest points with two distinctive patterns under different viewing conditions[12]. (2) In image recognition, the recording perspective in programming have the best recognizable and accurate rate, the accuracy rate of recognition would decrease with change of perspective (Diwadkar \& McNamara, 1997; Simons \& Wang, 1998; Waller, 2006). To solve this problem, we should set feature vectors around every interest points, which called descriptors. This descriptor can be used to make an interval between features; it also can reduce the noise and light to affect the accuracy rate of recognition. (3) Finally, recognizing the different descriptor based on distance arrange, in this way distance be defined to the difference of physical quantities, such as interval, area and proportion of the relative position.

So we can conclude interest points, descriptors and distance that play important role in pattern recognition. In addition, boundary shape is an important factor to recognize image by human vision. These four factors we called recognizable factors. When designers consider patterns design that can be recognized by computer vision, they can follow these factors and use some elements to structure a pattern, such as point, line and plate. Therefore, the main development of this study would structure a pattern systematically and research a reasonable process of pattern recognition.

\section{Methodology}

In this study, the experiment goals that we aim to establish a new method of pattern recognition for smart kitchen. This study use pattern recognition technology to operate interface components, the design of pattern must comply with the principle of the computer vision and have to make user feel pleasure when they use it. In order to explore the pattern that could be used in TUIs, the goals of experiment we expected to propose a serial of pattern design rules that could be recognized by algorithm. In 
related works we reviewed the basic rule between pattern design and computer vision, the experiment would extract the feature of pattern and establish a serial of pattern motif. The implementation of experiment would divide into two parts. At the first, we asked participants to share their experience and behavior in kitchen when they used kitchenware. After the focus group we can obtain some patterns drawn by participants, then we extract reasonable pattern design feature for systematic recognizable pattern. In order to confirm the pattern could be recognized effectively in various circumstances, we would use the program of recognition to test and evaluate it.

\subsection{Experiment 1: Using Focus Group to Establish the Feature of Pattern Design}

At the first we conducted focus group to discuss user behavior and problems in kitchen, then every participants considered how TUIs could use to solve these problems and mentioned their own viewpoints. Second parts we make participant draw the ideal pattern on paper. After all we collected the data of discussion to find out what problem could be solved by TUIs. While we collected drawing data to extract the features of pattern and classified these features into the four recognizable factors (interest points, descriptors, distance and boundary shape) to complete a systematical morphological chart of pattern.

\subsubsection{The Participants of Focus Group}

There were 7 male and 11 female university and graduate school students, age ranging from 19 to 24 (mean=21.06), served as participants in this experiment. These students all major in design and learned design more than 2 years. And they all have experience of cooking and using kitchen.

\subsubsection{Investigate Pattern Design by Focus Group}

The methodology that we use was participatory design to develop the pattern design. Focus group was used to gather participant concept for pattern design to help researcher consider the pattern design more deeply and gain the answer close to user further. One focus group divided into two parts, the first part we requested participant to share their experience use $5 \mathrm{w} 1 \mathrm{~h}$, such as when they were use the kitchenware, what problems they thought and so on. The second part we requested participants to think how to put the pattern on kitchenware and let them to draw their idea.

At first, researcher would outline the purpose of the group and the rules in this focus group. Because in this research we assumed the smart system set in kitchen, every participant received 10 sketch papers that size same to bowl, plate and cup. Then we asked participants to write down ideas and drew their concepts for pattern design. In two hours, focus group follows below process to discuss three pattern design topic: 
- The researcher asked participants to introduce themselves and talk their experience on using dinnerware, before using the topic guide to generate discussion.

- Cutlery of dinnerware (bowls, plates, and cups, dinnerware, cooking utensils, pans, spice jars, knives and kettles) displayed on table that can be close watched and touched. Every participant wrote down and presented their ideas about dinnerware using 5W1H: WHO, WHY, WHEN, WHERE, WHAT, HOW.

- Write down personal view by imaging if a pattern was on kitchenware for recognition, how they would work.

- Present the image that was written down. Drew any ideas on sketch paper.

Finally every participant discussed their sketch with each other and explored what structure of graphic is the most beautiful and appropriate. In this study we hold the focus group three times. Every time discuss different topic of kitchenware. So that we can get vaster information about pattern user behavior and pattern design.

\subsubsection{Data Analysis}

We used thematic analysis to analyze the focus group data. Thematic analysis is proposed by Aronson [13], which use reasonable steps to observe and clarify the situational context of cultural interaction to analyze qualitative data. Thematic analysis is usually used in qualitative research and focuses on examining themes within data. So the flow of data analysis was followed the process" entirety- part entirety" to explain context data. Thematic represented the elements which appear frequently in content, including argument, sentence and the meaning under situational context. Thematic analysis is the process of reproduction of these topics. Reviewing interview context data, which data repeated to appear constantly that is the common theme we want to explore. Then we could confirm the theme form qualitative data.

\subsection{Experiment 2: Evaluation and Confirmation the Database of Feature of Pattern}

According to the result of related works, we integrated the main recognizable factors of computer vision and the feature of pattern. The main factors respectively were boundary shape, interest points, descriptors and distance. We expected to use these four basic factors to explore how to make up a pattern systematically that could be recognized practically by algorithm. After we integrated the sketches that participant drew from focus group, we sorted the feature of pattern and classified the feature depend on four recognizable factors into morphological chart as the database of pattern structure. Then we referred to the result of thematic analysis and used the morphological chart to design new patterns. In the process of pattern design we makes each recognizable factors be used at least once.

In order to test our design pattern, we put them into a control condition and there set up two cameras, one set on the user's top, the other set on the user's side. In the environment we used two cameras to capture the pattern on an object in one minute with action including handling, stationary, tilt, movement and placement. A serial of action conducted three times pattern detecting. The process of detecting technology 
can be divided into following steps: (1) Given a search range (Mean Shift Algorithm), (2)capture Surf feature, (3) feature intensity defined (a line represent feature similarity between current image and model image), (4) whether feature intensity is greater than $0.68 \%$, (5) Feature matching amount is greater than 4 lines. If SURF algorithm did not detect the pattern that correspond to the model pattern, searching area would conduct to the center of camera vision and extended the $10 \%$ of searching area to search again. If SURF algorithm detected the pattern program would capture the image then updated the position of target pattern to re-determine the search range. Through the rate of algorithm recognition we could evaluate the rationality of pattern design and realized the design shortcomings. As a result, this technology could widely use to design appropriate pattern to attach on object that can be recognized in smart environment and accomplish the need of smart home in future.

\section{$4 \quad$ Result}

\subsection{Focus Group Result}

The focus groups generated rich information and a number of themes relating to behavior at kitchen and pattern design. The focus group classified the topic to nine serials to discuss, respectively bowls, plates, cups, dinnerware, cooking utensils, pans, spice jars, knives and kettles. At the first we used thematic analysis to review the first experiment data, the result we got users' problems and habitual behaviors if they would use pattern recognition in kitchen. The most common problem was hand or food probably covers up the pattern on kitchenware. In addition the participants also frequently proposed kitchen tools always change angle due to manipulative rotation, it probably resulted the difficulty of pattern recognition. Some participants suggested that we can put the pattern on spice jars not only can be recognized by algorithm but also can remind user what kind of spice contain in jars. The participants also suggested we can make annular pattern attach to the kitchenware side and utilize repeated pattern to avoid the problem that due to handheld cover pattern to lead to the difficulty of recognition. The position of pattern could place on the grip end of part. These suggestions all we have to pay much attention to consider when we implement the pattern recognition system in kitchen.

The second step we sorted out pattern design data, the result shown on figure 1. The most frequent pattern that the participant drew down was plane geometry and hollow annular graphics. The pattern design consisted of simple and repeated elements not only conform to the structure method of traditional pattern but also correspond with the recognizable factors on computer vision. We analyzed and got the feature of pattern, the process shown as Figure2. At first researcher distinguished boundary shape and found main interest points, then defined distance between two interest points. Finally, we defined descriptors around an interest point. We followed this principle to distinguish and capture recognizable factors on every pattern to establish a morphological chart as Table 1. 


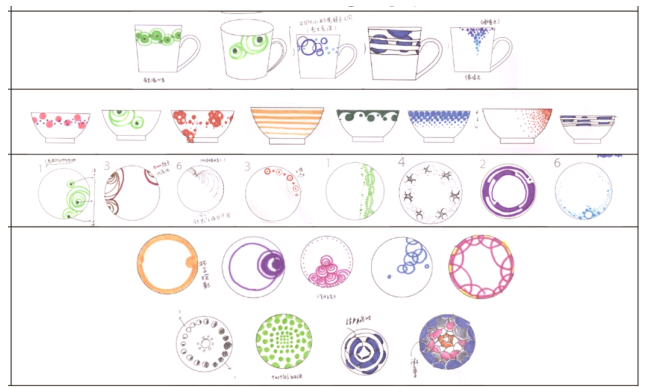

Fig. 1. The sketch was drawn from participant

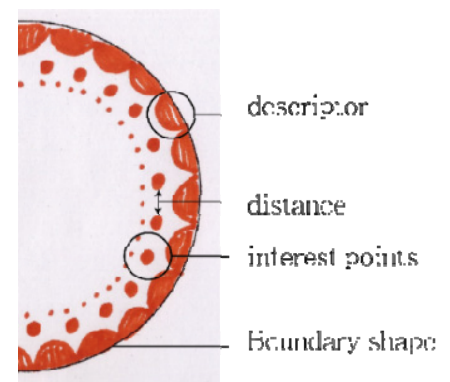

Fig. 2. The process of capture feature

In morphological chart, recognizable factors respectively were interest points, descriptors, distance and boundary shape. Boundary shape can be divided into square, triangle, circle and hexagon. Interest points referred to the specific position of feature when SURF algorithm detect pattern to recognize it. Descriptors were the feature around interest points to assist SURF algorithm locate interest points precisely, it can change its position with a different arrangement. Distance was the physical difference between interest points, such as interval, colors and proportion of area.

Table 1. Recognizable pattern morphological chart

\begin{tabular}{cccc}
\hline $\begin{array}{c}\text { Boundary } \\
\text { shape }\end{array}$ & Interest point & Distance & Descriptor \\
\hline Square & Dot & Area ratio & Shape \\
Triangle & Cross & Interval & Extend \\
Circle & T cross & Color & Cross \\
Hexagon & & & Array \\
& & & Replicate \\
\hline
\end{tabular}




\subsection{Recognition Test}

After we established the morphological chart, we used it to design patterns aimed to test how the rate of recognition that was detected by computer vision. In this study we designed two patterns depend on morphological chart, the process of design shown on Figure3. Then we placed pattern on a control condition to test the rate of recognition by SURF algorithm, the condition shown on Figure 4.

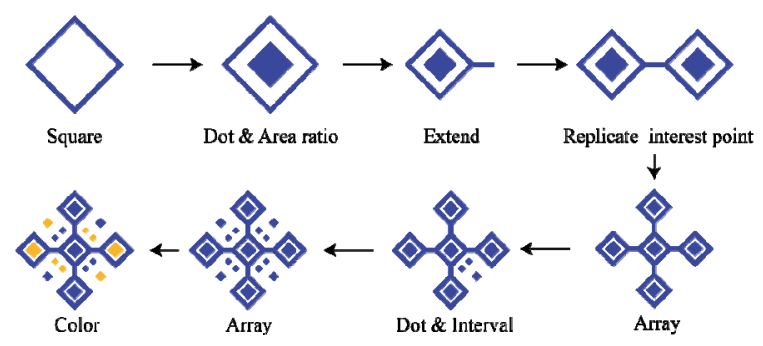

Fig. 3. Process of Pattern Design

Because of Image recognition standard rate was formulate by International Organization for Standardization(ISO) that have to exceed 66.7\% [14]. The recognition condition of SURF algorithm was shown on Figure 5. At first we placed the pattern model into the SURF algorithm, after started to search pattern in environment SURF algorithm would capture similar feature to compare with pattern model. Figure 5 shown if SURF algorithm captured corresponding feature, it would show black lines to connect between model pattern and reality image. The recognition rate of Red-bluecolor pattern averaged to be detected that was 37 times per minute among three operation and the average correct recognition rate was $92 \%$, it exceed ISO standard rate. However, the recognition rate of yellow -blue-color pattern averaged to be detected that was 35 times per minute among three operations but the average correct recognition rate was $0 \%$. It failed recognition that can be attributed to the influence of ambient light. When the algorithm processed computing, it converted image to grayscale to correspond to model pattern through computing the similarity of contrast. However, yellow has low contrast and the influence of ambient light, when SURF algorithm converted it to grayscale that is nearly white. Therefore, yellow -blue-color pattern cannot be recognized by SURF algorithm. To sum up, the recognizable factors were recognized specifically by SURF algorithm, except for color that greatly be influenced by ambient light.

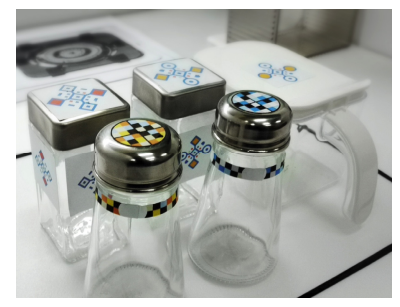

Fig. 4. Pattern testing on environment 


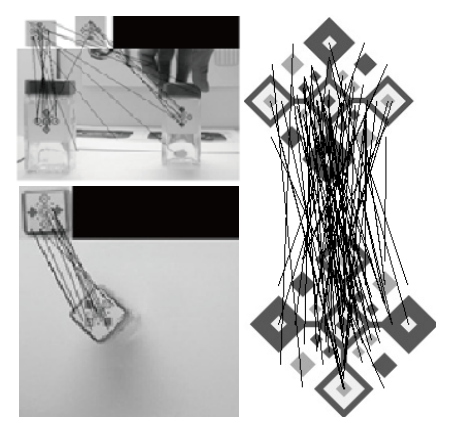

Fig. 5. Pattern was recognized

\section{Discussion}

According to the experiment result, the features of pattern which design with morphological chart were recognized successfully. In this study we confirmed a systematical method to design pattern and make the pattern of image recognition more aesthetic. Hence we expect to research and develop TUIs continually that based on the technology of pattern recognition in the future. However, there are some limitations in term of pattern recognition, (1) Recognizing objects is greatly affected by ambient lighting, pattern applied to TUIs that need sufficient ambient light and lights selection and configuration must be carefully arranged. (2)The harmonize colors of pattern should select higher-contrast colors to avoid failing recognition. (3) The position of pattern should consider carefully, because pattern on object probably be covered by hand or food.

The recognition of Computer vision usually are non-contextual, non-time sequence and without a cultural identity [15]. But human cognition is affected greatly by perceiving context and the background of culture. The main challenge of pattern recognition is that reduce the difference between computer vision and human cognition. Moreover, we conduct to research what kind of feedback can be used to respond to operation that complete with pattern recognition and how to reasonably design pattern to make operational object convey information to users.

\section{Conclusion}

As a result, we can expect to widely apply this technology to household items in the environment of smart home. In the future works we expect to use pattern recognition to make the operation of TUIs more intuitive and further conform users need and experience. Further we expand the usability of TUI, make interaction more natural, the concept of operation is more clearly. According to develop systematic pattern design method, we expect to achieve the object recognition system innovation in the future. 


\section{References}

1. Ishii, H., Ullmer, B.: Tangible bits: towards seamless interfaces between people, bits and atoms. In: Proceedings of the ACM SIGCHI Conference on Human factors in computing systems, pp. 234-241. ACM, Atlanta (1997)

2. van der Vlist, B.J.J., et al.: Semantic connections: Exploring and manipulating connections in smart spaces. In: 2010 IEEE Symposium on Computers and Communications (ISCC) (2010)

3. Lee, J.Y., et al.: Virtual and Pervasive Smart Home Services Using Tangible Mixed Reality. In: International Symposium on Parallel and Distributed Processing with Applications, ISPA 2008 (2008)

4. Shaer, O., Hornecker, E.: Tangible User Interfaces: Past, Present, and Future Directions. Found. Trends Hum.-Comput. Interact. 3, 1-137 (2010)

5. Garber, L.: Tangible User Interfaces: Technology You Can Touch. Computer 45(6), 15-18 (2012)

6. $\mathrm{Xu}, \mathrm{D}$., et al.: Designing and testing a tangible interface prototype. In: Proceedings of the 6th International Conference on Interaction Design and Children, pp. 25-28. ACM, Aalborg (2007)

7. Drori, T., Rinott, M.: Pixel Materiali: a system for creating and understanding pixel animations. In: Proceedings of the 6th International Conference on Interaction Design and Children, pp. 157-160. ACM, Aalborg (2007)

8. Shin, D.-H., Jung, J., Chang, B.-H.: The psychology behind QR codes: User experience perspective. Computers in Human Behavior 28(4), 1417-1426 (2012)

9. Grillo, A., et al.: High Capacity Colored Two Dimensional codes. In: Proceedings of the 2010 International Multiconference on Computer Science and Information Technology, IMCSIT (2010)

10. Bay, H., Tuytelaars, T., Van Gool, L.: SURF: Speeded up robust features. In: Leonardis, A., Bischof, H., Pinz, A. (eds.) ECCV 2006, Part I. LNCS, vol. 3951, pp. 404-417. Springer, Heidelberg (2006)

11. Xubing, Z., Hirai, S., Penglin, Z.: SURF and Spatial Association Correspondence applied in extraction and matching of feature points from MR images of deformed tissues. In: 2010 IEEE International Conference on Robotics and Biomimetics, ROBIO (2010)

12. Bay, H., et al.: Speeded-Up Robust Features (SURF). Computer Vision and Image Understanding 110(3), 346-359 (2008)

13. Aronson, J.: A Pragmatic View of Thematic Analysis. The Quality Report 2(1), 1989-1991 (1994)

14. Foster, J.J.: Standardizing Public Information Symbols: Proposals for a Simpler Procedure. Information Design Journal 6(2), 161-168 (1990)

15. Linhares, A., Chada, D.M.: What is the nature of the mind's pattern-recognition process? New Ideas in Psychology (2012) 